\title{
Determination of characteristics of anomalous light scattering in the gradient solutions using electronic speckle-pattern interferometry control of concentration distribution
}

\author{
${ }^{1}$ Bulavin L. A., ${ }^{1}$ GotsulskiyV. Ya. and ${ }^{2}$ Popov A. Yu. \\ ${ }^{1}$ Taras Shevchenko Kyiv National University \\ ${ }^{2}$ Scientific-Research Institute of Physics, I. I. Mechnikov Odesa National \\ University, popov-niif@onu.edu.ua
}

Received: 15.08 .2014

\begin{abstract}
We suggest a method for monitoring concentration dependences of the light scattering occurring in gradient solutions prepared by diffusion in the gravitational field. In this method, an electronic speckle-pattern interferometry (ESPI) approach is employed for non-contact measurements of spatial distribution of the solution concentrations. The method is applied to water-glycerol solutions. It has enabled us to study, in a simple way, the anomalous scattering peaks hardly observable with common techniques. The data obtained using our method are well reproducible and reliable.
\end{abstract}

Keywords: anomalous light scattering, aqueous solutions, electronic speckle pattern interferometry

PACS: 42.25.Fx, 42.25.Hz, 42.60.By, 61.20.Gy

UDC: $535.36,535.417,532.74,681.787 .7$

\section{Introduction}

Anomalous low-concentration scattering of light in aqueous solutions of alcohols is a very interesting phenomenon, which has still not been explained in terms of thermodynamic approach [1-6]. The effect is generally understood as an additional, when compared to the classic concentration scattering, anomalous low-concentration maximum of the light scattering, for which the scattering coefficient can be an order of magnitude higher than the normal one. A generalized characteristic of the light scattering intensity for the aqueous solutions of alcohols is displayed in Fig. 1 (see Refs. [1-3]). Here curve 1 corresponds to the additional low-concentration scattering peak, whose absolute value varies considerably for different alcohols, whereas the dotted line shows the dependence of the light scattering intensity as calculated from the thermodynamics of solutions based on the Gibbs potential [1]. It is this behaviour rather than the anomalous peaks themselves that is often observed experimentally.

Many authors have considered this phenomenon to be a model one, and so its investigations can contribute much to developing a theory of structural phase transitions in liquids, especially in those located near singular points [1-5]. Notice that experimental studies of the phenomenon are complicated by the fact that its manifestations strongly depend on the concentration, temperature and 'prehistory' of the solutions. What is important, the solutions achieve their thermodynamic equilibrium very slowly (the relaxation times are sometimes measured by tens of hours), and the equilibrium can be violated even by shaking. This leads to essential discrepancies among different experimental data, or even to their irreproducibility [2]. 


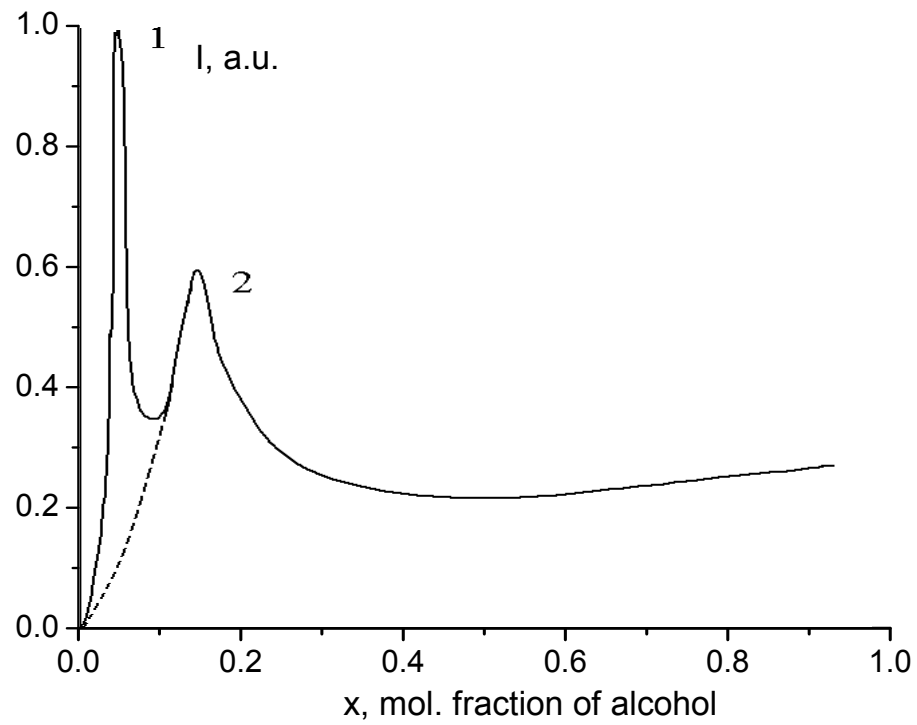

Fig. 1. Normalized light scattering intensity $I$ observed in wateralcohol solutions: curves 1 and 2 represent respectively an anomalous light scattering peak and a usual peak (i.e., a result of concentration fluctuations). Dashed line shows a scatteredlight intensity dependence expected theoretically.

In this work we propose a new method that facilitates studies of the above phenomenon, a method for monitoring concentration dependences of the light scattering in the gradient solutions prepared by diffusion in the gravitational field. We employ a non-contact, non-invasive laser electronic speckle pattern interferometry (ESPI) as a remote-sensing technique for determining the spatial distributions of the solution concentrations.

\section{Formulation of the problem}

Experimental studies of the anomalous light scattering with traditional methods face a number of difficulties. The main problem is strong dependence of the scattered light intensity on such factors as the concentration and the temperature of solution, as well as its 'prehistory' and mechanical stability. In the most of previous investigations, not all of these factors have been controlled simultaneously, and still less with a sufficient accuracy. This has resulted in the fact that some researchers could not observe the phenomenon under interest at all [1].

Issuing from these points, we conclude that an 'ideal' device for studying temporal and temperature dependences of the anomalous light scattering should enable observing and measuring the light scattering simultaneously for a number of cells containing solutions with different precisely prepared concentrations. However, since the concentrations in different cells must differ by very small amounts, the number of the cells must be large enough, e.g. dozens or even hundreds. It is thus clear that any practical implementation of such a device is technically challenging.

\section{The essence of the method}

We have developed and implemented a new method for the concentration studies of the light scattering in gradient solutions, which are prepared by mutual diffusion of components in the gravitational field. Under the condition of unlimited solubility of its components, the range of the solution concentrations can vary from 0 to $100 \%$. Then, instead of a discrete set of concentrations, a researcher can see a 'spatial scan' of concentrations in the volume of a single solution, which is maintained at a given temperature. The advantage of this method is that peaks of the anomalous scattering (if any) are always observable. The difficulty of the method is a need in accurate determination of the concentration peak positions. 
The necessity for experimental determination of the spatial distribution of concentrations comes from the fact that the diffusion coefficient is not constant. This has been demonstrated by measuring the characteristic times of collective motions of micro-inhomogeneities with a correlation spectroscopy technique [7-9]. Consequently, one cannot ensure that the concentration at each point of the solution can be found when basing only on the calculations of the mutual diffusion. Therefore the spatial distribution of the concentration must be measured experimentally, remotely and repeatedly for a long time. These requirements can be satisfied by phase-sensitive optical techniques, since the solution concentration is unambiguously (though not linearly) linked to the refractive index.

The most precise methods for the refractive index measurements are laser interferometric and holographic techniques (see, e.g., Ref. [10] where application of multi-beam interferometry for the investigation of diffusion processes in water-glycerol systems has been described). When dealing with such interferometric procedures, the size of a measuring cell must be very small. In Ref. [10] it has been $300 \mu \mathrm{m}$ and the above procedures are often unacceptable for larger cells. In our case the cell size was much larger, near $30 \mathrm{~cm}$. For these cell sizes, holographic controlling methods could be applied [11], but their implementation is very complex.

To solve this problem, we use a rather simple and flexible laser ESPI method, which is also known as a phase-modulated speckle interferometry. It is close to the methods of digital holography, which are now widely used in holographic microscopy. Unlike the latter methods, the laser ESPI method allows for investigating not only microscopic but macroscopic objects and, moreover, it does not involve expensive or exclusive equipment. In fact, the top size of the objects under test is limited only by the coherence length of a laser used in experiment.

In frame of the ESPI method, a laser beam testing an object has a speckle structure. The result of its superposition with a reference beam is a resulting speckle wave, at each point of which the intensity of light varies with changing reference wave phase. The key point of the ESPI measurement procedure is CCD-based detection of the light intensity distribution (referred to as a specklegram) in the resulting speckle wave at different phase shifts of the reference wave. The shifts used commonly are $0, \pi / 2$ and $\pi$, or $3 \pi / 2$ for more accurate measurements $[12,13]$.

Therefore, a set of patterns is detected under the condition that the phase distribution of the object wave must be constant during the whole procedure. If the correlation processing of a set of specklegrams detected at the beginning of measurements is carried out, one can determine the initial phase distribution in the object wave (i.e., a phase portrait). A comparison of the initial phase portrait with the current one, which is determined at a selected time moment, yields a picture of phase correlations, a so-called phasegram, which is similar to interferograms in a doubleexposure holographic interferometry. Further mathematical processing of those phasegrams can also be carried out using the algorithms developed for holographic interferometry.

\subsection{Experimental device}

Schematic diagram of our device is shown in Fig. 2. Here B1 denotes a unit for measuring the scattering distribution. It includes a laser L1 and a cell 1 with a gradient solution. An ESPI unit B2 for concentration distribution measurements comprises a laser L2, lenses 2 and 3, a scattering diffuser 4 , semi-transparent mirrors 5 , a mirror 6 , and an adjustable mirror 7 driven by computercontrolled piezoceramic 8 . A unit B3 for recording and controlling consists of a camcorder 9, a computer 10, and a controller 11. In fact, our device combines two devices designed for performing different tasks. 


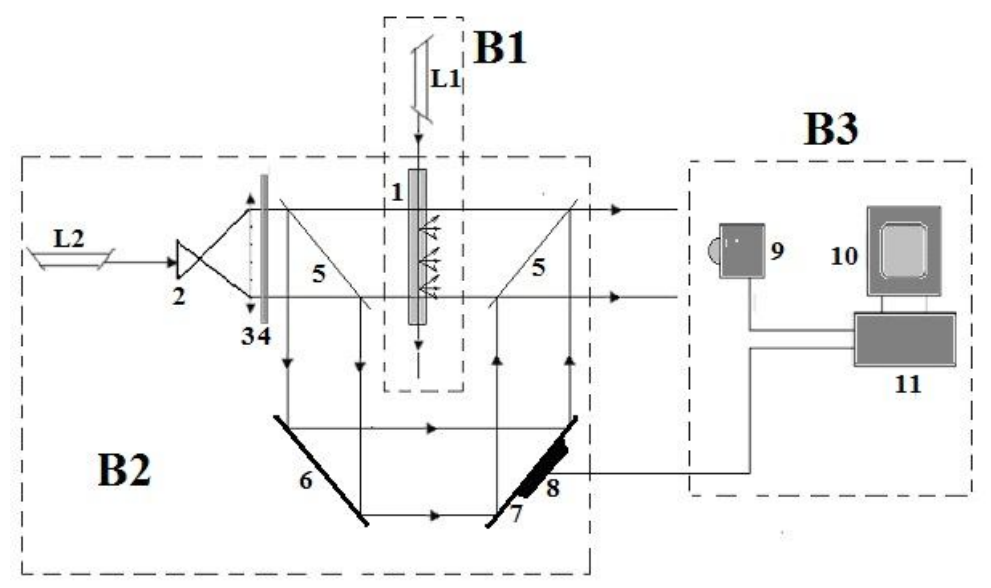

Fig. 2. Experimental setup for studying light scattering and determining the refractive index and the corresponding concentration of solution contained in a cell.

The unit B1 serves for observation of light scattering in a measuring cell that manifests a vertical gradient of concentration. It allows observing the light scattering from all the points along the path of laser beam L1 inside a solution with variable concentration. The unit B2 determines the concentration of this solution at any point in the cell. During the measurements, the both units can operate either simultaneously (if the radiation wavelength of the laser L2 is sufficiently separated from that of the laser L1) or alternately. To avoid confusion, further on we will call the beam of the laser 1 passing through the measuring cell as a probe beam and that of the laser 2 as a testing one (see Fig. 3). Finally, the unit B3 is general, being employed by the both units B1 and B2. It includes a low-noise CCD camera, a computer, a controller, and a set of original controlling and accounting programs united by a common interface. The measurements are performed automatically and a long-term monitoring regime is possible, too.

\subsection{Operation of the units}

Unit 1. We have prepared our solutions using diffusion in the gravitational field. Initially, the two chemically pure components of a system are placed into a cell. The cell represents vertically elongated prisms where a higher-density component is located at the bottom. After some time, a vertical spatial distribution of the concentration is formed as a result of diffusion. The probe beam (i.e. that of the laser L1) traverses through the cell along the vertical symmetry axis, i.e. along the concentration gradient (from top to bottom - see Fig. 2 and Fig. 3), and the light scattered in the perpendicular direction from a certain vertical 'slice' of the cell is being detected. Then one can get dependence of the scattering light intensity on the solution concentration. Here stray light reflections from the phase boundaries are excluded owing to immersion and an absorbing optical trap located at the cell bottom.

Unit 2. A difficulty of the present method is a necessity of accurate non-invasive determination of the solution concentration at each point of the sell, which can be achieved only with the optical methods. We have chosen an ESPI version of the method suggested in Ref. [12] as a high-precision approach for carrying out that task. Earlier it has been applied at the Research Institute of Physics, I. I. Mechnikov Odesa National University, Ukraine, for studying small mechanical displacements and strains, analysing phase structures of biological microobjects [13], etc. The method determines refractive index changes in the whole volume of the measuring cell 
with no scanning. Since the refractive index and the material density are unambiguously linked with each other, one can assess, in a real-time regime, the spatial distribution of the solution concentration in the cell via its phasegram.

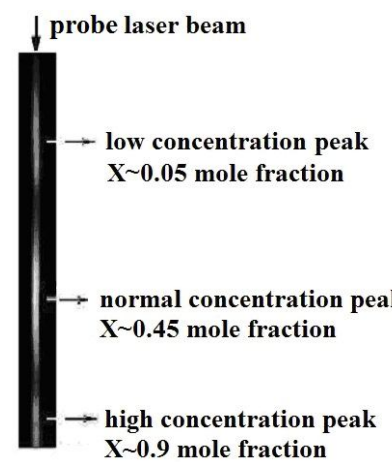

A

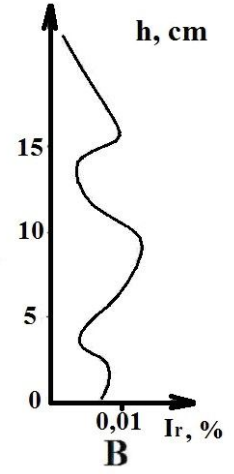

B

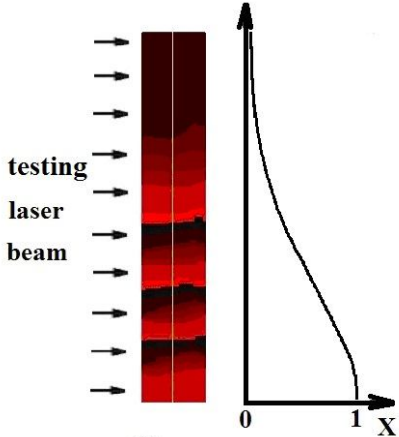

C

D

Fig. 3. (A) Spatial distribution of light scattered in the cell with pure glycerol at the bottom, clean water at the top, and gradient concentration changes in between, as detected photographically; (B) Plot of relative scattered light intensity $l_{r}$; (C) ESPI phasegram showing a spatial variation of testing-light phase proportional to the glycerol concentration changes; (D) Glycerol mole fraction $X$ calculated basing on the phasegram represented in panel (C).

The phasegrams (see Fig. 2) are obtained in the following way. With the aid of a coherent light source L2 (we use a He-Ne laser with the power of $50 \mathrm{~mW}$ ), and a system of lens 2 and 3, a wide monochromatic beam with plane front is formed. It falls onto a scattering diffuser 4 , after which the wave front acquires a speckle structure. The resulting speckle wave is divided by a semi-transparent mirror 5 into two beams, an object and reference ones. While passing through a measuring cell 1 , the object beam acquires some spatially inhomogeneous phase shift which correlates with the spatial distribution of solution concentration in the cell. A mirror 7 driven by a piezoceramic 8 modulates the speckle-field phase in the reference beam according to signals originated from a controller-synchronizer 11.

The object and reference beams are superimposed by a semi-transparent mirror 5 . The resulting speckle patterns (specklegrams) recorded by a CCD camera 9 are further processed by a computer 10. Frames are detected four times, with the relevant reference-wave phase shifts being equal to $0, \pi / 2, \pi$ and $3 \pi / 2$. The correlation-based processing of the resulting sets of specklegrams allows us to construct a phase portrait of the object. If necessary, the number of phase steps can be increased, thus increasing the measuring accuracy.

A methodology of the ESPI version used in this work employs the two following stages (see also Ref. [12]). The initial phase portrait is calculated from a set of specklegrams recorded in the temperature-stabilized measuring cell filled with clean water before the glycerol is introduced; the second phase portrait is derived from another set recorded at some selected moments after the glycerol is introduced. The cross-correlation processing of the two phase portraits, the initial and current ones, results in phase-correlation pictures (phasegrams). The latter represent the changes in the probing laser beam phase having happened between the two measurements. As mentioned above, these phasegrams are similar to interferograms usual in both classical and holographic interferometry. The important advantage here is that the 'direction' of the phase changes (increase or decrease) can be determined unambiguously (see Fig. 4). 


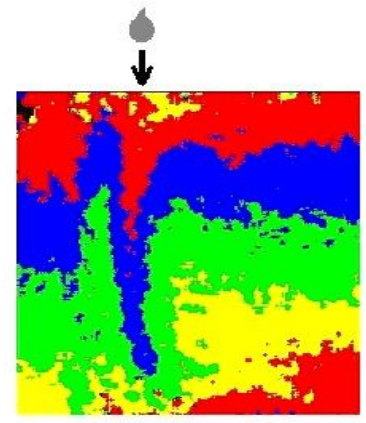

$\mathbf{A}$

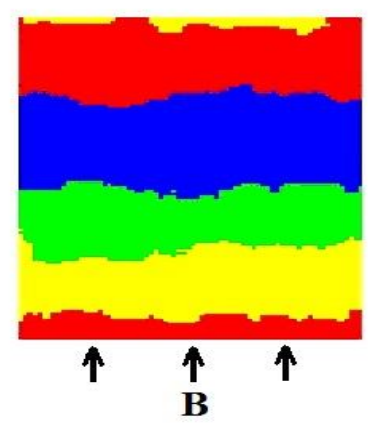

Fig. 4. ESPI phasegrams obtained 30 minutes after a drop of glycerol is introduced into the solution from the top (panel A) and after glycerol is injected from the bottom (panel B). Phase variations are indicated by colours: (blue) $0-\pi / 2$, (green) $\pi / 2-\pi$, (yellow) $\pi-3 \pi / 2$, and (red) $3 \pi / 2-2 \pi$.

The frequency of equi-phase lines in the phasegrams is proportional to the gradient of the solution concentration. We use the two types of graphic representations of the phasegrams: a fourcolor one for visual perception (see Fig. 4 and Fig. 5) and a monochrome gradient representation (see Fig. 3 and Fig. 5) which is convenient for further numerical processing.

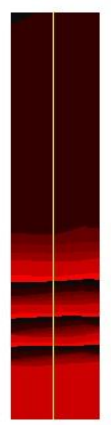

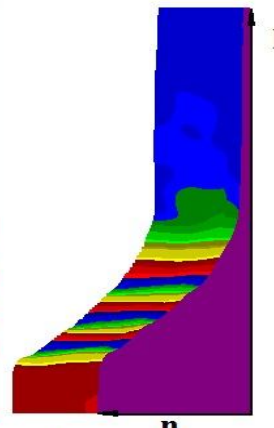

$\mathbf{A}$

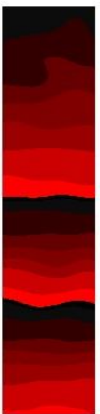

B

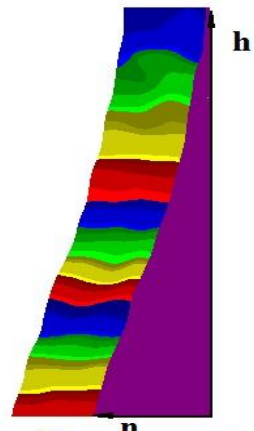

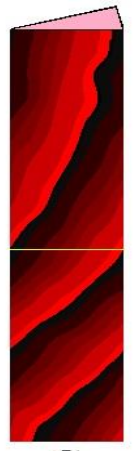

C

Fig. 5. The same phasegrams as in Fig. $3 \mathrm{C}$, along with the corresponding spatial distributions of refractive index $n$ along the vertical axis: $(A)$ and $(B)$ the cell of a rectangular shape, with the results obtained (A) $1 \mathrm{~h}$ and (B) $3 \mathrm{~h}$ after injecting glycerol; (C) the same as in panel (B) but for the case of wedge-like cell.

Fig. 4 proves extremely high sensitivity of the ESPI method to variations in the solution concentration and, at the same moment, the importance of employing a correct method for preparation of the solution. In the case of Fig. $3 \mathrm{~A}$, glycerol is introduced into the solution by drops from the top. Then the traces of the drops are clearly distinguishable for a long time, and the concentration distribution in the cell turns out to be rather chaotic. This is why in our subsequent experiments we have used the other method for injecting glycerol through the channels at the bottom of the measuring cell (see Fig. 4B).

\section{Experimental results and analyses of phasegrams}

\subsection{Scattering light intensity measurements (Unit 1)}

As a test sample, we have selected the aqueous solution of glycerol. Fig. 3 shows the results for the scattered light intensity. In particular, Fig. 3A displays a snapshot of the light scattered in a water-glycerol solution, with the spatial concentration of glycerol increasing from top to bottom. Here the probe laser beam passes along the symmetry axis of the system. The scattered light 
intensity is determined using a special image processing. Notice also that accumulation of video images during the experiment eliminates the noise. In Fig. 3B, the corresponding plot of the relative scattered light intensity $I_{r}$ is shown ( $I r=I_{s} / I_{i}$, with $I_{s}$ and $I_{i}$ being the intensities of respectively the scattered light and the probe beam). Three light scattering peaks can be simultaneously observed: the normal (classic) concentration peak, the anomalous lowconcentration one, and the anomalous high-concentration peak. Although the latter has been predicted theoretically [14], it has still not been experimentally detected in the earlier studies.

In Fig. 3B, the anomalous scattering peaks appear to be very broad because of a small vertical gradient of the glycerol concentration in the corresponding regions (see Fig. 3D). However, this allows one to investigate the concentration dependence of the light scattering with maximal accuracy, especially in the regions of the lowest and highest concentrations, which are of the greatest interest in this study. After converting the data to the absolute concentration values (see Fig. 6), we get a plot very similar to that displayed in Fig.1.

A question of correct identification is very important here: Are the additional light scattering peaks detected by our gradient method the same as those observed under stationary conditions? Although the solutions studied with the gradient method can in many ways be considered as quasistationary, there are still implicit hydrodynamic flows due to the diffusion. Regarding this difference, it is not evident whether the origins of the low-concentration peaks shown in Fig. 3A, $\mathrm{B}$ are the same as those described in Refs. [1-3].

However, at the moment we consider this question as premature, at least due to the fact that there are no generally accepted ideas about the physical reasons for the anomalous scattering even in the most thoroughly studied stationary conditions, not to mention the gradient conditions. It is safe to say that, in the both cases, the anomalous scattering peaks are similar. This refers to preconditions of their occurrence, the concentration positions, and the temperature dependences (see Fig. 6). Of course, there are important questions: (i) Are there any differences in these cases? and (ii) Are they significant? These problems will be elucidated in the following studies.

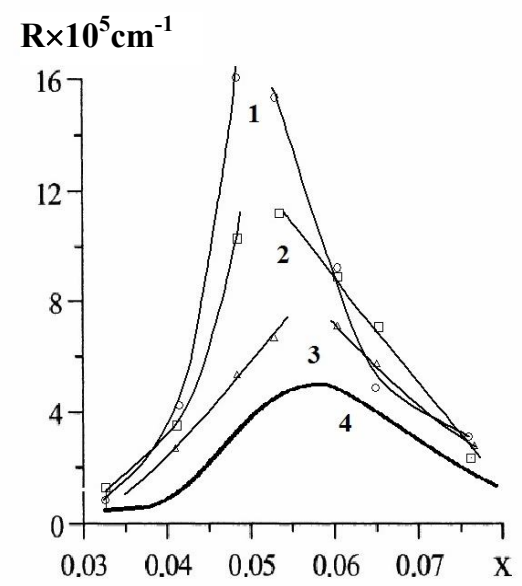

Fig. 6. Additional low-concentration peaks of light scattering in aqueous glycerol solution as obtained from dependences of the scattering coefficient $R$ upon the mole fraction $X$ of glycerol at different temperatures: (1) $T=3^{\circ} \mathrm{C}$; (2) $T=5^{\circ} \mathrm{C}$; (3) $T=10^{\circ} \mathrm{C}$, and (4) $T=20^{\circ} \mathrm{C}$. Curves 1,2 and 3 represent data for the solutions with discrete concentrations, and curve 4 is obtained with the gradient method.

\subsection{Determination of glycerol concentration distribution in the measuring cell}

A theoretical possibility of finding the glycerol concentration $X$ at each point of the cell arises since the latter quantity is directly associated with the refractive index $n$. However, the dependence $X(n)$ is nonlinear because any real solutions are not ideal, and it must be determined with 
independent refractometric methods. Fig. 7 shows a dependence of the refractive index of waterglycerol solutions reported by one of the largest producers of chemicals, a company "Dow". Having such a calibration data, one can reduce the problem of evaluating the concentration $X$ to determining the refractive index $n$ of the solution at each point of the measuring cell. With our method, it is carried out in the two variants corresponding to the measuring cells of rectangular and wedge-like shapes (see Fig. 5).

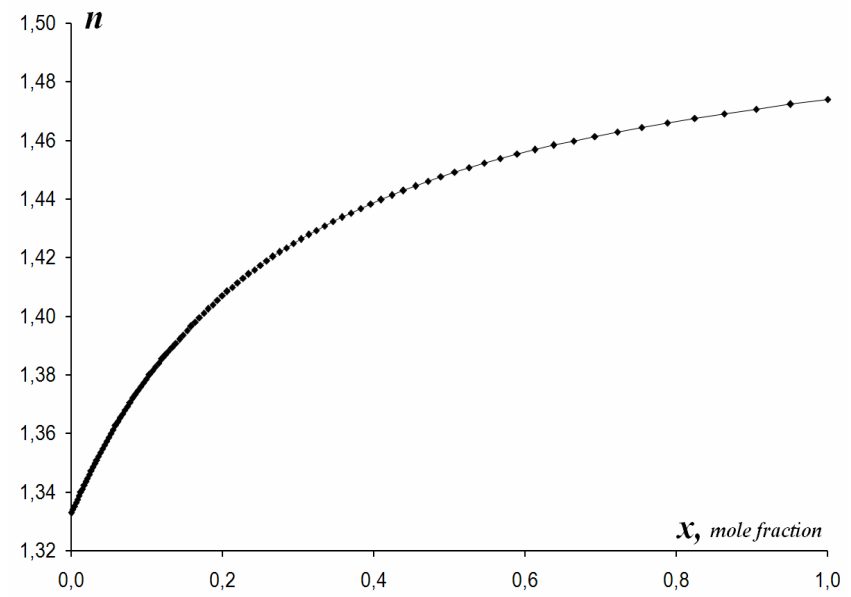

Fig. 7. Refractive index $n$ of aqueousglycerol solutions versus mole glycerol fraction $X$ as obtained at $t=20^{\circ} \mathrm{C}$ according to the company "Dow".

\subsubsection{The case of rectangular cell}

Variations $\Delta n$ of the refractive index at a given point of the cell are associated with the phase changes $\Delta \Psi$ for the testing laser beam at this point:

$$
\Delta \Psi=\frac{2 \pi \Delta n d}{\lambda} .
$$

Here $d$ is the 'thickness' of the liquid along the light ray at a given point and $\lambda$ the wavelength of the testing laser irradiation.

The ESPI phasegrams enable determining the phase variations along the vertical section, thus yielding the parameter $\Delta n$. However, one must have a reference point to learn the $n$ value. At the early stages of diffusion (see Fig. 5A), while the layers of pure glycerol (on the bottom) and water (on the top) coexist, the concentration $X$ at each point can be determined uniquely. On the contrary, if there are no reference points (see Fig. 5B), this becomes impossible from the analysis of a single phasegram. The task can nevertheless be accomplished if one analyzes a set of phasegrams obtained at different times during the transition from the case A to the case B and, in such a manner, determines temporal variations of $n$ at a given point.

\subsubsection{Accuracy of the concentration measurements}

The measuring accuracy depends on the accuracy of measurements of the phase and the cell thickness, with the latter governing the overall phase difference between pure water and glycerol. In the phasegram shown in Fig. 7, this full phase difference is about $\sim 8 \pi$. Then the $n$ varies from 1.33 to 1.47 and the concentration $X$ from 0 to 1 .

The ESPI method applied in this work measures the phase changes with the accuracy of up to $\pi / 100$. Then, in the ideal case, the accuracy for measuring $n$ can be as high as $18 \times 10^{-5}$. After taking into account the digit capacity of the CCD camera used for detecting specklegrams (640× 480 in our case), the accuracy is slightly reduced, down to $23 \times 10^{-5}$. Although this is good enough, 
the accuracy can be increased by at least an order of magnitude if thicker cells and cameras with higher resolutions are employed. Thus, the accuracy of evaluating the concentration $X$ could rise almost up to the accuracy of the calibration data (see Fig. 7).

Notice that while implementing our method in practice, we have had to overcome a number of significant technical difficulties. The measuring cells containing liquid samples must meet very high standards. In case of the rectangular measuring cells, the thickness of the liquid layer $d$ should be constant over the entire area of the cell. Any 'distortions' in $d$ would lead to distorted phase structure of the testing beam and would therefore decrease the accuracy. It is difficult to fulfil these demands with the cells of large sizes. However, deviations in the thickness can be corrected with software. This requires measuring a spatial distribution of changes in the thickness $\Delta d$ of liquid throughout the area of the measuring cell. The latter can be implemented directly in our device by using the same ESPI method. Namely, the cell should be filled, one by one, with a number of liquids having different, though precisely known in advance, refractive indices. The case of a pair of pure water and pure glycerol is shown in Fig. 8.

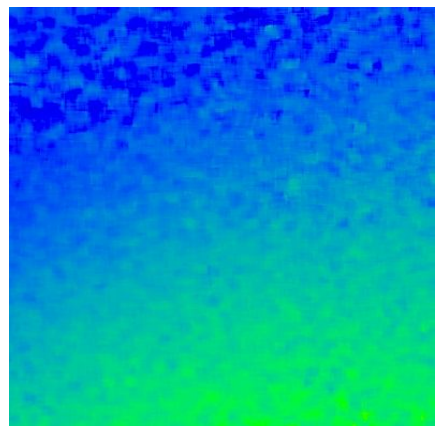

Fig. 8. Test phasegram obtained for a rectangular cell $\left(20 \times 20 \times 1 \mathrm{~cm}^{3}\right)$ by replacing pure water with pure glycerol.

It is obvious from the phasegram that the phase changes approximately by $\pi / 2$ and the changes are smooth along the vertical direction. Hence, the thickness of the layer contained in the cell also varies smoothly (by $\sim 1 \mu \mathrm{m}$ ). Besides, one can observe small-scale fluctuations of about $\sim 0.1 \mu \mathrm{m}$. This data should be properly taken into account when processing the experimental phasegrams. It is worthwhile to note that such a large cell width is not necessary. We have chosen it merely to avoid the edge effects, whereas the measurements have been performed in the central, i.e. the least perturbed, area. As shown in the present study, the boundary perturbation is not very significant, and the cell width can be reduced to about $2 \mathrm{~cm}$ with no loss of accuracy. This would greatly facilitate the temperature-related studies because the temperature has to be stabilized in a significantly smaller volume.

\subsubsection{The case of wedge-like cell}

When wedge-like sells are used, one can dispense with the reference points for unambiguous phase evaluation. In this case $\Delta \Psi$ in the cross section equals to

$$
\Delta \Psi=\frac{2 \pi n \Delta d}{\lambda}
$$

where $\Delta d=\alpha x$ implies the variable cell thickness, $x$ the horizontal transverse coordinate, and $\alpha$ the angular coefficient of the wedge. When the calibrated thickness change $\Delta d$ is known for every cross-section point of the cell, the above relationship enables determining the refractive index $n$ of the solution at a given height $h$, with no reference concentration points (see Fig. 5C). Then the following requirements for the rectangular cell remain important: the thickness of the liquid in the 
wedge-shaped cells must be maximally stable in the vertical direction and vary linearly in the horizontal one.

There arises an extra difficulty: to analyze the data, one must be sure that the solution concentration along a selected horizontal cross-sectional line remains constant, which may not hold true if the rates of diffusion in the narrow and wide parts of the wedge differ from each other. Nevertheless, this condition is easily controlled: if the concentration is indeed constant, the phase variation along the horizontal cross-sectional line has to be linear.

At present, we have used only rectangular cells in any practical work. The advantages and disadvantages of using the rectangular and wedge-like cells for various researches require further consideration.

\subsection{Advantages of our method}

Finally, we emphasize the main positive features of the experimental techniques developed in this study: (i) Our method is very simple and easy to use in practice, allowing to obtain simultaneously a complete scan of dependences of the scattered-light intensity on the concentration, and (ii) The data obtained with our method are reproduced well and so reveal high reliability, unlike the methods employed previously.

\section{Conclusions}

Basing on the results of the present work, one can summarize the following features and capabilities of our methods:

1. Observations of concentration dependences for the solutions with concentration gradients, as suggested in this study, should certainly replace the common measurements which require preparations of many samples with different concentrations. Indeed, while using our method, one simultaneously obtains a complete scan of the concentration-scattering dependence. As a consequence, we have successfully identified the normal-concentration maximums of light scattering studied previously and the anomalous low-concentration scattering maximums. In addition, we have revealed the anomalous high-concentration maximum predicted in Ref. [14].

2. Besides of the light scattering dynamics, our method allows for exploring dynamics of the concentration dependences of various physical quantities associated with both temporal and temperature variations.

3. Application of the ESPI method has resulted in reliable data for the changes in the spatial distribution of concentration in our samples. The relevant experimental data are in good agreement with earlier theoretical calculations of inter-diffusion process for a pair of semiinfinite material media, as well as with the results available in the earlier experimental studies [10].

4. Our method employs computer-based approaches that automate the measurements and enable permanent monitoring without a researcher present. In the future, we plan to fulfil precise experimental determination of the absolute concentration values at necessary time moments. A need in such measurements stems from highly complicated time-temperature dependences of the characteristic times of collective motion appearing in the objects under monitoring [7].

\section{Acknowledgements}

This work was partly supported by the Ministry of Education and Science of Ukraine (Project No 494).

Ukr. J. Phys. Opt. 2014, Volume 15, Issue 4 


\section{References}

1. Vuks M F. Light Scattering in gases, liquids and solutions. Leningrad: Izd. LGU, 1977.

2. Anisimov M A. Critical phenomena in liquids and liquid crystals. Moscow: Nauka, 1987.

3. Subramanian D and Anisimov M A, 2011. Resolving the mystery of aqueous solutions of tertiary butyl alcohol. J. Phys. Chem. B. 115: 9179-9183.

4. Lanshina L V and Dakar G M, 2003. Molecular light scattering by clathrate-like structures in the water-2-butanol-acetone system. Ross. Zhurn. Fiz. Khim. 77: 948-951.

5. Bunkin N F, Gorelik V S, Sabirov L M, Semenov D I and Khaidarov Kh S, 2010. Frequency shift of Rayleigh line fine structure components in a water solution of 4-methylpyridine as a function of temperature, concentration, and light scattering angle. Quant. Electron. 40: 817821.

6. Chechko V Eu, Lokotosh T V, Malomuzh N P, Zaremba V G and Gotsul'sku V Ya, 2003. Clusterization and anomalies of fluctuations in alcohol solutions of low concentrations. J. Phys. Stud. 7: 175-183.

7. Chechko V E, Gotsulskiy V Ya and Zaremba V G, 2003. On the nature of relaxation processes in dilute water-glycerol solutions. J. Mol. Liquids. 105/2-3: 211-214.

8. Gotsulsky V Y, Malomuzh N P and Chechko V E, 2013. Features of the temperature and concentration dependences of the contraction of aqueous solutions of ethanol. Ross. Zhurn. Fiz. Khim. A. 87: 1638-1644.

9. Chechko VE, Gotsulsky V Ya and Malomuzh M P, 2013. Peculiar points in the phase diagram of the water-alcohol solutions. Cond. Matt. Phys. 16: 1-9.

10. Abramov A Yu, Ryabukho V P and Shipovskaya A B, 2010. Laser interferometry method in studying the diffusion process in the glycerol-water system. Vestn. Saratov Gos. Univer., Ser. Fiz. 10: 35-41.

11. Jones R and Wykes C. Holographic and speckle interferometry. Cambridge: Cambridge University Press, 1989.

12. Smyntyna V A, Tyurin O V, Popov Yu A and Zhukovsky V K. Method of phase modulated speckle interferometry to measure the object wave phase change. Patent UA No 7343, Bull. No 6, 2005.

13. Popov A Yu, Popova N A, Tyurin A V and Grimblatov V, 2013. Effects of speckle-like laser irradiation on growth of bacteria in vitro. Proc. SPIE. 8569. Mechanisms for Low-Light Therapy VIII, 85690C.

14. Chechko VEu, 2011. Peculiarities of anomalous light scattering in solutions with strong hydrogen bonds. PhD thesis. I. I. Mechnikov Odesa National University. 164 p.

Bulavin L. A., GotsulskiyV. Ya. and Popov A. Yu. 2014. Determination of characteristics of anomalous light scattering in the gradient solutions using electronic speckle-pattern interferometry control of concentration distribution. Ukr.J.Phys.Opt. 15: 216 - 226.

Анотація. У роботі запропоновано метод моніторингу концентраційних залежностей розсіяння світла в градієнтних розчинах, приготовлених дифузією в гравітаційному полі. $У$ рамках иього методу для безконтактного вимірювання просторового розподілу концентрації розчинів вжито електронну спекл-інтерферометрію. Метод застосовано у вивченні розчину вода-гліцерол для спрощення досліджень аномальних піків розсіяння, які важко спостерігати звичайними методами. Дані, одержані за нашим методом, відтворюються 3 достатньою точністю. 